IJMMS 29:3 (2002) 125-131

PII. S0161171202011626

http://ijmms.hindawi.com

(c) Hindawi Publishing Corp.

\title{
ALMOST SURE CENTRAL LIMIT THEOREMS FOR STRONGLY MIXING AND ASSOCIATED RANDOM VARIABLES
}

\author{
KHURELBAATAR GONCHIGDANZAN
}

Received 24 January 2001 and in revised form 9 June 2001

We prove an almost sure central limit theorem (ASCLT) for strongly mixing sequence of random variables with a slightly slow mixing rate $\alpha(n)=O\left((\log \log n)^{-1-\delta}\right)$. We also show that ASCLT holds for an associated sequence of random variables without a stationarity assumption.

2000 Mathematics Subject Classification: 60F05, 60F15.

1. Introduction and main results. The almost sure central limit theorem (ASCLT) has been first introduced independently by Schatte [11] and Brosamler [4]. Since then, many interesting results have been discovered in this field. For further results on ASCLT we refer to Berkes [1].

An interesting direction is to prove ASCLT for weakly dependent cases, namely, $\alpha$, $\rho, \phi$-mixing, and associated random variables. Among the results in this direction we refer to Peligrad and Shao [9], Hurelbaatar [5], and Matuła [7].

Let $X_{1}, X_{2}, \ldots$ be a sequence of random variables on some probability space $\left(\Omega, \mathscr{F}_{,}, P\right)$, and let $\sigma_{a}^{b}$ be the $\sigma$-algebra generated by the random variables $X_{a}, X_{a+1}, \ldots, X_{b}$. For any two $\sigma$-algebras $\mathscr{A}, \mathscr{B} \subset \mathscr{F}$, define

$$
\alpha(\mathscr{A}, \mathscr{B})=\sup \{|\mathbf{P}(A B)-\mathbf{P}(A) \mathbf{P}(B)| ; A \in \mathscr{A}, B \in \mathscr{B}\}
$$

and put

$$
\alpha(n)=\sup _{k \geq 1} \alpha\left(\sigma_{1}^{k}, \sigma_{k+n}^{\infty}\right)
$$

The sequence $X_{1}, X_{2}, \ldots$ is called strongly mixing if $\alpha(n) \rightarrow 0$ as $n \rightarrow \infty$.

A sequence of random variables $X_{1}, X_{2}, \ldots$ is called associated if for every $n \geq 1$ and any coordinatewise increasing functions $f, g: \mathbb{R}^{n} \rightarrow \mathbb{R}^{1}$,

$$
\operatorname{Cov}\left(f\left(X_{1}, X_{2}, \ldots, X_{n}\right), g\left(X_{1}, X_{2}, \ldots, X_{n}\right)\right) \geq 0
$$

whenever the covariance is defined.

We set $S_{n}=X_{1}+X_{2}+\cdots+X_{n}$ and the notation $a_{n} \ll b_{n}$ means $a_{n}=O\left(b_{n}\right)$. The function $\mathbf{I}_{A}(\cdot)$ denotes an indicator function on the set $A$.

Peligrad and Shao [9] proved ASCLT for stationary Gaussian sequences as well as stationary associated random variables. Their main results are as follows. 
THEOREM 1.1 (see [9, Theorem 3]). Let $X_{1}, X_{2}, \ldots$ be a stationary $\alpha$-mixing sequence with $\mathbf{E} X_{1}=0, \mathbf{E} X_{1}^{2}<\infty, a_{n}^{2}=\mathbf{E} S_{n}^{2} \rightarrow \infty$ as $n \rightarrow \infty$ and $\alpha(n) \ll \log ^{-\gamma} n$, for some $\gamma>0$. Assume that

$$
\frac{S_{n}}{a_{n}} \stackrel{\mathscr{D}}{\longrightarrow} N(0,1) \quad \text { as } n \longrightarrow \infty
$$

Then ASCLT holds, that is,

$$
\lim _{n \rightarrow \infty} \frac{1}{\log n} \sum_{k=1}^{n} \frac{1}{k} \mathbf{I}_{A}\left(\frac{S_{k}}{a_{k}}\right)=\frac{1}{\sqrt{2 \pi}} \int_{A} e^{-t^{2} / 2} d t \quad \text { a.s. }
$$

for all Borel sets $A \subset \mathbb{R}$ with $\lambda(\partial A)=0$.

THEOREM 1.2 (see [9, Theorem 2]). Let $X_{1}, X_{2}, \ldots$ be a stationary associated sequence with $\mathbf{E} X_{1}=0$, and $\sum_{k=1}^{\infty} \mathrm{E} X_{1} X_{k}<\infty$. Then (1.5) holds.

In this paper, we prove almost sure limit theorems which generalize Theorems 1.1 and 1.2. We also show that under the stationarity assumption, Theorems 1.4 and 1.6 imply Theorems 1.1 and 1.2, respectively. For strong mixing we impose much slower mixing rate. Our main results are as follows.

THEOREM 1.3. Let $X_{1}, X_{2}, \ldots$ be a sequence of random variables with zero mean. Assume that

$$
\operatorname{Var}\left(\sum_{k=1}^{n} \frac{1}{k} f\left(\frac{S_{k}}{a_{k}}\right)\right) \ll(\log \log n)^{-1-\epsilon} \log ^{2} n
$$

for all bounded Lipschitz functions $f(x)$. Then (1.5) holds if and only if

$$
\lim _{n \rightarrow \infty} \frac{1}{\log n} \sum_{k=1}^{n} \frac{1}{k} \mathbf{P}\left(\frac{S_{k}}{a_{k}} \in A\right)=\frac{1}{\sqrt{2 \pi}} \int_{A} e^{-t^{2} / 2} d t
$$

for all Borel sets $A \subset \mathbb{R}$ with $\lambda(\partial A)=0$.

THEOREM 1.4. Let $X_{1}, X_{2}, \ldots$ be a strongly mixing sequence of random variables with mean zero, and let $a_{n}>0$ be a numerical sequence such that $\mathrm{E} S_{n}^{2} \leq a_{n}^{2}$ and for $n \geq k$

$$
\frac{a_{n}}{a_{k}} \geq\left(\frac{n}{k}\right)^{\gamma}, \quad \gamma>0 .
$$

Assume that

$$
\alpha(n) \ll(\log \log n)^{-1-\delta} .
$$

Then (1.5) and (1.7) are equivalent.

Berkes, Dehling, and Móri [3] gave an example of independent random variables such that (1.5) was satisfied, but (1.4) failed. This shows that the class of sequences satisfying ASCLT is larger than the class of sequences satisfying CLT. Berkes and Dehling [2] proved the equivalence of (1.5) and (1.7) for independent random variables not necessarily identically distributed, under mild technical condition on generalized 
moments of the partial sums. This result has been generalized by Hurelbaatar [5] for strongly mixing and associated random variables. In Theorem 1.4, concerning the limit distributional behavior of $S_{n} / a_{n}$, we require more restrictive moment condition than the one in Hurelbaatar [5] and Berkes and Dehling [2].

REMARK 1.5. For stationary sequence of $\alpha$-mixing random variables, it is known that $a_{n}=n L(n)$, where $L(n)$ is a slowly varying function at infinity, provided the central limit theorem holds (see [6, page 316]). By the representation theorem of slowly varying function, $L(s) / L(t) \gg(s / t)^{-\epsilon}$ for any $\epsilon>0$ and $s \geq t \geq n_{0}(\epsilon)$ and we see that condition (1.8) is satisfied for the $a_{n}$ and $\gamma=1 / 2-\epsilon$. Therefore, for stationary sequence of random variables Theorem 1.4 implies Theorem 1.1.

THEOREM 1.6. Let $X_{1}, X_{2}, \ldots$ be a sequence of associated random variables with mean zero satisfying

$$
u(n)<\infty
$$

for all $n \geq 1$ and where $u(n)=\sup _{k \geq 1} \sum_{j:|k-j| \geq n} \operatorname{Cov}\left(X_{k}, X_{j}\right)$.

Assume that (1.8) is satisfied for some $\gamma>0$ and

$$
\liminf _{k} \operatorname{Var} X_{k}>0 \text {. }
$$

Then (1.5) and (1.7) are equivalent.

REMARK 1.7. In stationary case, the assumption of Theorem 1.2 implies the one of Theorem 1.6. We can easily verify that

$$
\begin{gathered}
\sum_{k=1}^{\infty} \operatorname{Cov}\left(X_{1}, X_{k}\right)<\infty, \\
u(n)=\sup _{k \geq 1} \sum_{j:|k-j| \geq n} \operatorname{Cov}\left(X_{k}, X_{j}\right)<\infty
\end{gathered}
$$

are equivalent for a stationary sequence of associated random variables. It is well known that if (1.12) holds, then

$$
\lim _{n \rightarrow \infty} \frac{\operatorname{Var}\left(S_{n}\right)}{n}=\mathbf{E} X_{1}^{2}+2 \sum_{k=1}^{\infty} \mathbf{E} X_{1} X_{k} .
$$

Choosing $a_{k}^{2}$ as $\operatorname{Var}\left(S_{k}\right)$, we see that (1.8) is satisfied with $\gamma=1 / 2$. By Newman and Wright [8, Theorem 3], (1.4) is true under the assumption of Theorem 1.2 and implies (1.7). Thus Theorem 1.2 is a stationary case of Theorem 1.6.

\section{Proofs}

Proof of Theorem 1.3. It suffices to show that (see [2])

$$
\mu_{n}=\frac{1}{\log n} \sum_{k=1}^{n} \frac{1}{k} \xi_{k} \longrightarrow 0 \text { a.s. }
$$

for any bounded Lipschitz function $f$, where $\xi_{k}=f\left(S_{k} / a_{k}\right)-\mathbf{E} f\left(S_{k} / a_{k}\right)$. 
By (1.6) we have

$$
\begin{aligned}
\mathbf{E} \mu_{n}^{2} & =\mathbf{E}\left(\frac{1}{\log n} \sum_{k=1}^{n} \frac{1}{k} \xi_{k}\right)^{2}=\frac{1}{\log ^{2} n} \mathbf{E}\left(\sum_{k=1}^{n} \frac{1}{k} \xi_{k}\right)^{2} \\
& =\frac{1}{\log ^{2} n} \operatorname{Var}\left(\sum_{k=1}^{n} \frac{1}{k} f\left(\frac{S_{k}}{a_{k}}\right)\right) \ll(\log \log n)^{-1-\epsilon},
\end{aligned}
$$

and setting $n_{k}=\exp \left(\exp \left(k^{\gamma}\right)\right)$,

$$
\mathbf{E} \mu_{n_{k}}^{2} \ll\left(\left(\log \log n_{k}\right)^{-1-\epsilon}\right) \ll k^{-\gamma(1+\epsilon)} .
$$

Hence

$$
\sum_{k=1}^{\infty} \mathbf{E} \mu_{n_{k}}^{2}<\infty
$$

for any $\gamma>1 /(1+\epsilon)$.

By the well-known result that

$$
\sum_{k=1}^{\infty} \mathbf{E} X_{k}<\infty \quad \text { implies } \quad \sum_{k=1}^{\infty} X_{k}<\infty \quad \text { a.s., }
$$

we have

$$
\mu_{n_{k}} \rightarrow 0 \text { a.s. }
$$

It is easy to see that

$$
\left((1+k)^{\epsilon}-k^{\epsilon}\right) \longrightarrow 0 \text { as } k \longrightarrow \infty \text { for any } \epsilon<1,
$$

and thus

$$
\frac{\log n_{k+1}}{\log n_{k}}=e^{(1+k)^{\gamma}-k^{\gamma}} \longrightarrow 1 \quad \text { as } k \longrightarrow \infty
$$

Obviously, for any given $n$ there always exists $k$ such that $n_{k} \leq n \leq n_{k+1}$ and we have

$$
\begin{aligned}
\left|\mu_{n}\right| & \leq \frac{1}{\log n} \sum_{k=1}^{n} \frac{1}{k}\left|\xi_{k}\right| \leq \frac{1}{\log n_{k}} \sum_{k=1}^{n_{k}} \frac{1}{k}\left|\xi_{k}\right|+\frac{1}{\log n_{k}} \sum_{k=n_{k}}^{n_{k+1}} \frac{1}{k}\left|\xi_{k}\right| \\
& \ll\left|\mu_{n_{k}}\right|+\frac{1}{\log n_{k}}\left(\log n k+1-\log n_{k}\right) \ll\left|\mu_{n_{k}}\right|+\left(\frac{\log n_{k+1}}{\log n_{k}}-1\right) .
\end{aligned}
$$

It follows that

$$
\lim _{n \rightarrow \infty} \mu_{n}=0 \quad \text { a.s. }
$$


Proof of TheOrem 1.4. According to Theorem 1.3, it suffices to show that for all bounded Lipschitz,

$$
\operatorname{Var}\left(\frac{1}{\log n} \sum_{k=1}^{n} \frac{1}{k} f\left(\frac{S_{k}}{a_{k}}\right)\right) \ll(\log \log n)^{-1-\epsilon} .
$$

Here $\xi_{k}$ is the same variable as we defined in the proof of Theorem 1.3. For $l>2 k$, we have

$$
\begin{aligned}
\left|\mathbf{E}\left(\xi_{k} \xi_{l}\right)\right|= & \left|\operatorname{Cov}\left(f\left(\frac{S_{k}}{a_{k}}\right), f\left(\frac{S_{l}}{a_{l}}\right)\right)\right| \\
\leq & \left|\operatorname{Cov}\left(f\left(\frac{S_{k}}{a_{k}}\right), f\left(\frac{S_{l}}{a_{l}}\right)-f\left(\frac{S_{l}-S_{2 k}}{a_{l}}\right)\right)\right| \\
& +\left|\operatorname{Cov}\left(f\left(\frac{S_{k}}{a_{k}}\right), f\left(\frac{S_{l}-S_{2 k}}{a_{l}}\right)\right)\right| .
\end{aligned}
$$

Since $f$ is bounded, by [10, Theorem 1.1], we get

$$
\left|\operatorname{Cov}\left(f\left(\frac{S_{k}}{a_{k}}\right), f\left(\frac{S_{l}-S_{2 k}}{a_{l}}\right)\right)\right| \ll \alpha(k),
$$

and by Cauchy-Schwarz inequality, Lipschitz property of $f$, the facts that $\mathbf{E} S_{n}^{2} \leq a_{n}^{2}$, and by (1.8) we have

$$
\begin{aligned}
\left|\operatorname{Cov}\left(f\left(\frac{S_{k}}{a_{k}}\right), f\left(\frac{S_{l}}{a_{l}}\right)-f\left(\frac{S_{l}-S_{2 k}}{a_{l}}\right)\right)\right| \\
\ll \mathbf{E}\left(\frac{\left|S_{2 k}\right|}{a_{l}}\right) \ll\left(\mathbf{E}\left(\frac{S_{2 k}}{a_{l}}\right)^{2}\right)^{1 / 2} \ll \frac{a_{2 k}}{a_{l}} \ll\left(\frac{k}{l}\right)^{\gamma} .
\end{aligned}
$$

Noting that

$$
\begin{aligned}
\mathbf{E}\left(\sum_{k=1}^{n} \frac{1}{k} \xi_{k}\right)^{2} & \leq \sum_{k=1}^{n} \frac{1}{k^{2}} \mathbf{E}\left|\xi_{k}\right|^{2}+2 \sum_{\substack{1 \leq k<l \leq n \\
2 k \geq l}} \frac{\left|\mathbf{E}\left(\xi_{k} \xi_{l}\right)\right|}{k l}+2 \sum_{\substack{1 \leq k<l \leq n \\
2 k<l}} \frac{\left|\mathbf{E}\left(\xi_{k} \xi_{l}\right)\right|}{k l} \\
& =\mathbf{T}_{1}+\mathbf{T}_{2}+\mathbf{T}_{3}
\end{aligned}
$$

and since $\xi_{k}$ is bounded, we have the following estimations for the first two terms

$$
\mathbf{T}_{1} \ll \sum_{k=1}^{\infty} \frac{1}{k^{2}}<\infty, \quad \mathbf{T}_{2} \ll \sum_{k=1}^{n} \sum_{l=k+1}^{2 k} \frac{1}{k l} \ll \log n .
$$

By (2.12), (2.13), and (2.14) the third term is estimated as

$$
\mathbf{T}_{3} \ll \sum_{\substack{1 \leq k<l \leq n \\ 2 k<l}} \frac{1}{k l}\left(\frac{k}{l}\right)^{\gamma}+\sum_{\substack{1 \leq k<l \leq n \\ 2 k<l}} \frac{\alpha(k)}{k l}=\mathbf{T}_{31}+\mathbf{T}_{32},
$$


and furthermore

$$
\begin{aligned}
\mathbf{T}_{32} & \ll \sum_{l=2}^{n} \frac{1}{l} \sum_{k=1}^{l-1} \frac{\alpha(k)}{k} \ll \sum_{k=1}^{n} \frac{\alpha(k)}{k} \sum_{l=k}^{n} \frac{1}{l} \\
& \ll \sum_{k=1}^{n} \frac{\log k}{k(\log \log k)^{1+\delta}} \ll(\log \log n)^{-1-\delta} \log ^{2} n \\
\mathbf{T}_{31} & \leq \sum_{\substack{1 \leq k<l \leq n \\
2 k<l}} \frac{1}{k l}\left(\frac{k}{l}\right)^{\gamma} \ll \sum_{l=1}^{n} \frac{1}{l^{1+\gamma}} \sum_{k=1}^{l} \frac{1}{k^{1-\gamma}} \ll \sum_{l=1}^{n} \frac{1}{l} \ll \log n .
\end{aligned}
$$

Now it can be easily shown that (2.15), (2.16), (2.17), (2.18), and (2.19) together give (1.6) which is equivalent to (2.11).

Proof of Theorem 1.6. The general idea of the proof is similar to the method in the proof of Theorem 1.4. We will verify (2.11). From (2.15) we see that

$$
\begin{aligned}
\operatorname{Var}\left(\sum_{k=1}^{n} \frac{1}{k} f\left(\frac{S_{k}}{a_{k}}\right)\right) & =\mathbf{E}\left(\sum_{k=1}^{n} \frac{1}{k} \xi_{k}\right)^{2} \\
& \leq \sum_{k=1}^{n} \frac{1}{k^{2}} \mathbf{E}\left|\xi_{k}\right|^{2}+2 \sum_{\substack{1 \leq k<l \leq n \\
2 k \geq l}} \frac{\left|\mathbf{E}\left(\xi_{k} \xi_{l}\right)\right|}{k l}+2 \sum_{\substack{1 \leq k<l \leq n \\
2 k<l}} \frac{\left|\mathbf{E}\left(\xi_{k} \xi_{l}\right)\right|}{k l} \\
& =\mathbf{T}_{1}+\mathbf{T}_{2}+\mathbf{T}_{3},
\end{aligned}
$$

and moreover,

$$
\begin{aligned}
\mathbf{T}_{3}= & \sum_{\substack{1 \leq k<l \leq n \\
2 k<l}} \frac{\left|\mathbf{E}\left(\xi_{k} \xi_{l}\right)\right|}{k l}=\sum_{\substack{1 \leq k<l \leq n \\
2 k<l}} \frac{1}{k l}\left|\operatorname{Cov}\left(f\left(\frac{S_{k}}{a_{k}}\right), f\left(\frac{S_{l}}{a_{l}}\right)\right)\right| \\
\leq & \sum_{\substack{1 \leq k<l \leq n \\
2 k<l}} \frac{1}{k l}\left|\operatorname{Cov}\left(f\left(\frac{S_{k}}{a_{k}}\right), f\left(\frac{S_{l}}{a_{l}}\right)-f\left(\frac{S_{l}-S_{2 k}}{a_{l}}\right)\right)\right| \\
& +\sum_{\substack{1 \leq k<l \leq n \\
2 k<l}} \frac{1}{k l}\left|\operatorname{Cov}\left(f\left(\frac{S_{k}}{a_{k}}\right), f\left(\frac{S_{l}-S_{2 k}}{a_{l}}\right)\right)\right| \\
= & \mathbf{T}_{31}+\mathbf{T}_{32} .
\end{aligned}
$$

For a bounded Lipschitz function $f$, it is shown, by Peligrad and Shao [9], that

$$
\operatorname{Cov}\left(f\left(\frac{S_{k}}{a_{k}}\right), f\left(\frac{S_{l}-S_{2 k}}{a_{l}}\right)\right) \ll \operatorname{Cov}\left(\frac{S_{k}}{a_{k}}, \frac{S_{l}-S_{2 k}}{a_{l}}\right) .
$$

Hence

$$
\mathbf{T}_{32} \ll \sum_{\substack{1 \leq k<l \leq n \\ 2 k<l}} \frac{1}{k l}\left|\operatorname{Cov}\left(\frac{S_{k}}{a_{k}}, \frac{S_{l}-S_{2 k}}{a_{l}}\right)\right| \ll \sum_{\substack{1 \leq k<l \leq n \\ 2 k<l}} \frac{1}{k l} \frac{k u(k)}{a_{k} a_{l}} .
$$

For associated random variables, clearly

$$
\operatorname{Var} S_{n} \geq \sum_{k=1}^{n} \operatorname{Var} X_{k}^{2}
$$


By the assumption that $\mathbf{E} S_{n}^{2} \leq a_{n}^{2}$ and (1.11), we get

$$
a_{n}^{2} \gg \sum_{k=1}^{n} \operatorname{Var} X_{k}^{2} \gg n,
$$

therefore,

$$
\begin{aligned}
\mathbf{T}_{32} & \ll \sum_{\substack{1 \leq k<l \leq n \\
2 k<l}} \frac{k u(k)}{k^{3 / 2} l^{3 / 2}} \ll \sum_{k=1}^{n} \frac{u(k)}{k^{1 / 2}} \sum_{l=k}^{n} \frac{1}{l^{3 / 2}} \\
& \ll \sum_{k=1}^{n} \frac{\log k}{k(\log \log k)^{1+\delta}} \ll(\log \log n)^{-1-\delta} \log ^{2} n .
\end{aligned}
$$

By (2.14) and (2.19)

$$
\mathbf{T}_{31} \ll \log n,
$$

and (2.16), (2.26), and (2.27) follow (2.11).

Acknowledgements. I am very grateful to Prof. Magda Peligrad for her helpful discussion. Also I would like to thank the referees for their helpful comments and remarks. This work was supported by a Research Fellowship at the University of Cincinnati.

\section{REFERENCES}

[1] I. Berkes, Results and problems related to the pointwise central limit theorem, Asymptotic Methods in Probability and Statistics (Ottawa, ON, 1997), North-Holland, Amsterdam, 1998, pp. 59-96.

[2] I. Berkes and H. Dehling, Some limit theorems in log density, Ann. Probab. 21 (1993), no. 3, 1640-1670.

[3] I. Berkes, H. Dehling, and T. F. Móri, Counterexamples related to the a.s. central limit theorem, Studia Sci. Math. Hungar. 26 (1991), no. 2-3, 153-164.

[4] G. A. Brosamler, An almost everywhere central limit theorem, Math. Proc. Cambridge Philos. Soc. 104 (1988), no. 3, 561-574.

[5] G. Hurelbaatar, Almost sure limit theorems for dependent random variables, Studia Sci. Math. Hungar. 33 (1997), no. 1-3, 167-175.

[6] I. A. Ibragimov and Y. V. Linnik, Independent and Stationary Sequences of Random Variables, Wolters-Noordhoff, Groningen, 1971.

[7] P. Matuła, On the almost sure central limit theorem for associated random variables, Probab. Math. Statist. 18 (1998), no. 2, 411-416.

[8] C. M. Newman and A. L. Wright, An invariance principle for certain dependent sequences, Ann. Probab. 9 (1981), no. 4, 671-675.

[9] M. Peligrad and Q. M. Shao, A note on the almost sure central limit theorem for weakly dependent random variables, Statist. Probab. Lett. 22 (1995), no. 2, 131-136.

[10] E. Rio, Covariance inequalities for strongly mixing processes, Ann. Inst. H. Poincaré Probab. Statist. 29 (1993), no. 4, 587-597.

[11] P. Schatte, On strong versions of the central limit theorem, Math. Nachr. 137 (1988), 249256.

KhurelbaAtar Gonchigdanzan: Department of Mathematical SCIENCES, University of Cincinnati, CincinnATI, OH 45221-0025, USA

E-mail address: hur1ee@math.uc.edu 


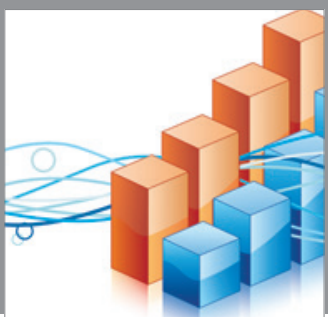

Advances in

Operations Research

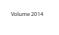

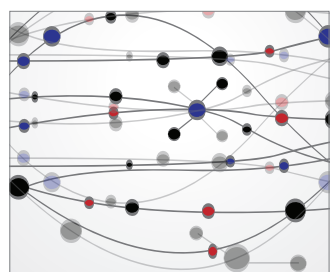

\section{The Scientific} World Journal
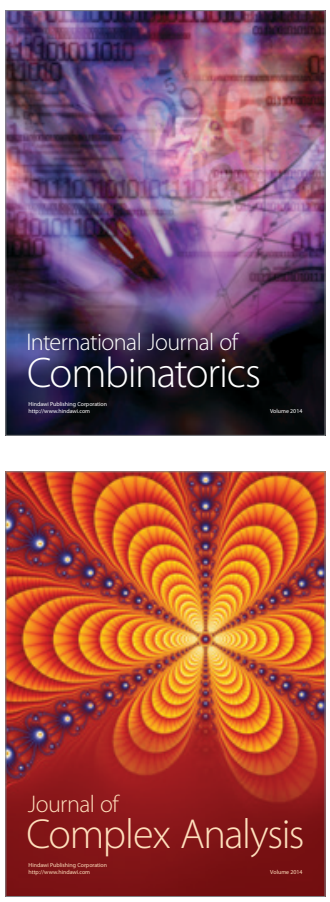

International Journal of

Mathematics and

Mathematical

Sciences
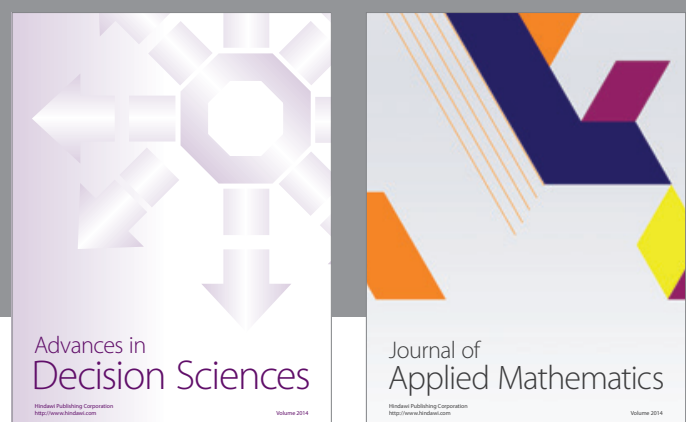

Journal of

Applied Mathematics
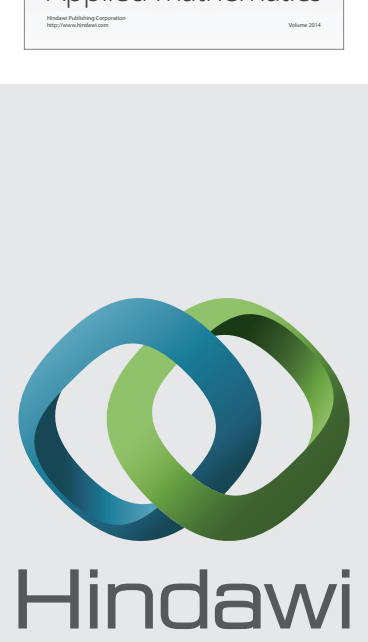

Submit your manuscripts at http://www.hindawi.com
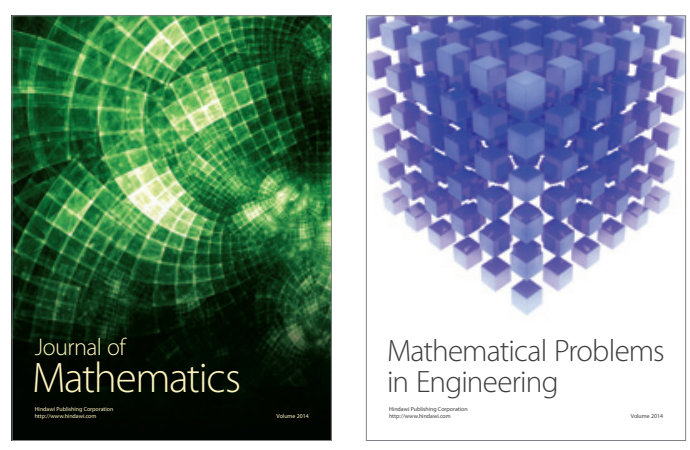

Mathematical Problems in Engineering
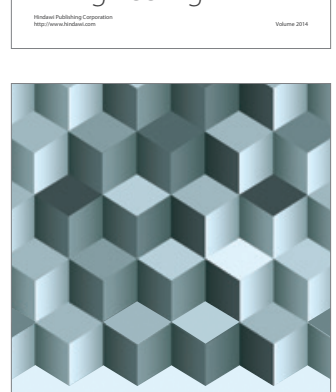

Journal of

Function Spaces
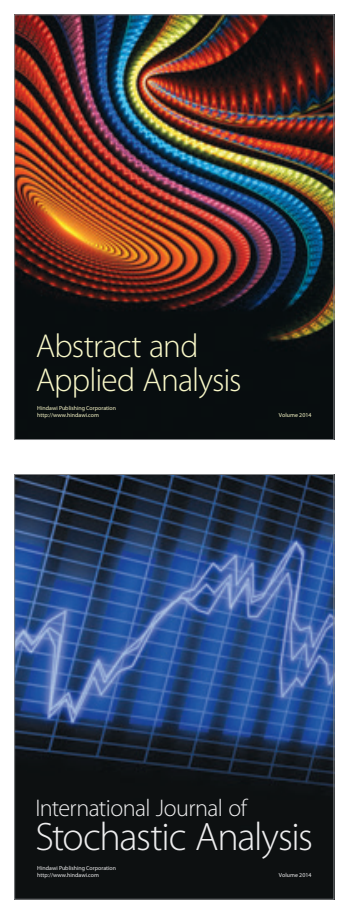

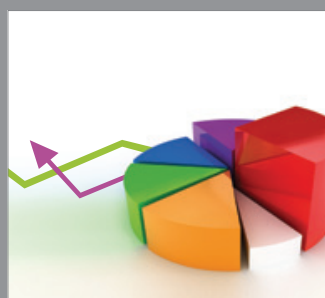

ournal of

Probability and Statistics

Promensencen
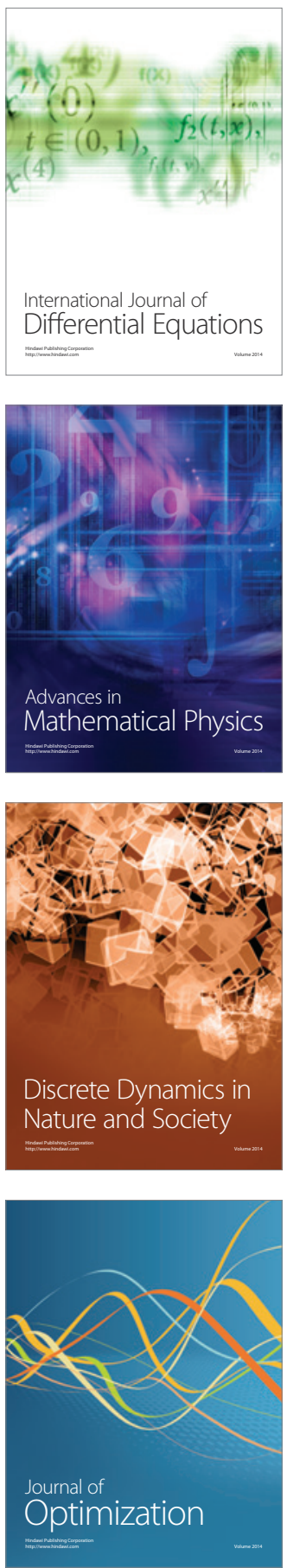